\title{
CPG-based Control of a Turtle-like Underwater Vehicle
}

\author{
Keehong Seo, Soon-Jo Chung, Jean-Jacques E. Slotine
}

\begin{abstract}
We present a new bio-inspired control strategy for an autonomous underwater vehicle by constructing coupled nonlinear oscillators, similar to the animal central pattern generators (CPGs). Using contraction theory, we show that the network of oscillators globally converges to a specific pattern of oscillation. We experimentally validate the proposed control law using a turtle-like underwater vehicle, whose fin actuators successfully exhibit a pattern that resembles the motion of fore limbs of a swimming sea turtle. In order to further fulfill the potential of the CPG-based control, we propose to feed back the actuator states to the coupled oscillators, thereby achieving not only the synchronization of the oscillators, but also the synchronization of actual foil states. Such a closed-loop version of CPGs makes the controller more robust and practical in the presence of external disturbances.
\end{abstract}

\section{INTRODUCTION}

Biologically inspired approaches to locomotion have been studied in robotics to develop robots like snake [1], fish [2], salamander [3], and so on. The flexibility and adaptability of the bio-inspired mechanism in dealing with the environment has been discussed in the literature, especially in the context of an alternative solution to traditional means of locomotion such as wheels and propellers. To control these biologically inspired types of robotic locomotion, a plausible approach is to mimic or get inspired from animal central pattern generators (CPGs), leading to modular designs.

A CPG is a neuronal network that exists in animal spinal cord to govern the locomotion [4], [5], [6]. CPGs are believed to relieve the burdens of the central nerve system in controlling the locomotion. In other words, animals walk or run even without paying much attention to the periodic movement of their legs. The CPG system has inspired many robotic researchers since it can reduce the control bandwidth required from the main controller to its actuators.

In engineering applications, the dominant approach is using governing oscillators to represent the neurons in the $\mathrm{CPG}$ network and the outputs of the oscillators are used to generate torque inputs or reference signals for servos. Some [7], [8] use feedback from sensors to adapt phase of the governing oscillators while others [9], [10], [11] use open-loop approach

K. Seo was in the Nonlinear Systems Laboratory, Massachusetts Institute of Technology, Cambridge, MA 02139; currently in the Department of Aerospace Engineering, Iowa State University, Ames, IA 50011, Email: kseo@iastate.edu

S. -J. Chung is in the Aerospace Robotics Laboratory, the Department of Aerospace Engineering, Iowa State University, Ames, IA 50011, Email: sjchung@iastate.edu

J. -J. E. Slotine is in the Nonlinear Systems Laboratory, the Department of Mechanical Engineering, Massachusetts Institute of Technology, Cambridge, MA 02139, Email: jjs@mit.edu without the feedback from sensors to the oscillators, depending on the complexity of the application.

For many types of robots, the actuation for locomotion is essentially an oscillatory motion. It is also true for our testbed of interest, a turtle-like autonomous underwater vehicle (AUV) as introduced in [12]. Its fluidic maneuvers are controlled by the roll and pitch motion of its four fins that mimic the fore limbs of a sea turtle. The roll and pitch motions used for the operation of vehicle in [12] was basically harmonic oscillations.

Our new approach for biologically inspired control simplifies the conventional CPG-based control to establish a robust coordination of the actual fin motions. While our method is based on limit cycle oscillators, we induce the oscillation to emerge by feeding the fin states directly back to the fin actuators. Independent from our study, the emerging oscillation in the close-loop has been studied in a neuro-mechanical system as in [13]. The resulting oscillator exhibits a limit cycle behavior and the oscillation properties can be adjusted by modifying the system parameters. Once the oscillation of the fin motion is established, we can let the motions synchronize among the fins by using diffusive coupling of velocities from other fins. The rolling motion of fins synchronize themselves and the pitch motion in a fin synchronize with its rolling motion with a 90-degree phase difference.

One advantage of the proposed approach over the openloop CPG method is that we enforce the synchronization of the actuators directly while the open-loop CPG approach enforces only the synchronization of the reference inputs to the actuators. In the open-loop approach, if some disturbance was applied to a fin, the reference input from CPG still produces sinusoidal waves regardless of the current state of the fin, thereby potentially inducing large errors at some moment, which could in turn result in unnecessarily large control effort for recovery. In contrast, if the fin itself is already a selfsustaining oscillator, it does not need the clock-like reference input. In case of the recovery from the disturbance, it can return to its limit cycle from where it was, which would require less control effort than tracking a time-specific reference trajectory. During the recovery phase, the synchronization with other fin is simultaneously achieved through the diffusive velocity couplings, as we prove theoretically and demonstrate experimentally. In essence, the proposed approach serves as a flexible means to recover from disturbances, which is an important characteristic of the robustness to rapidly changing environments.

The paper is organized as follows. Section II describes the 


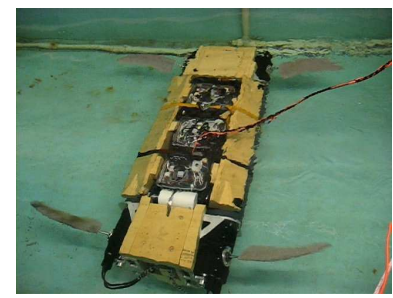

(a)

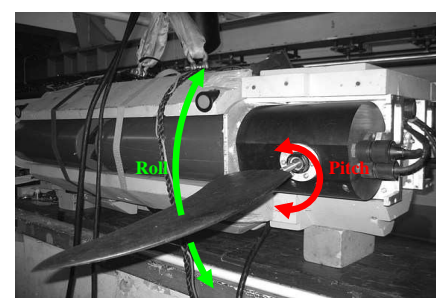

(b)
Fig. 1. "The biomimetic flapping foil autonomous underwater vehicle (BFFAUV) was conceived as a test platform and proof of concept for the use of flapping foils as the sole source of propulsion and maneuvering forces in an underwater vehicle," quoting [12]

turtle-like AUV as the testbed for the experimental validation. In section III, we introduce previous CPG-based control approaches. In section IV, the new CPG-based control strategy is proposed and its performance of synchronization is discussed. In section $\mathrm{V}$, we present experimental results by implementing the proposed approach in the AUV.

\section{The Bio-inspired Underwater Vehicle System}

As the test platform of our approach for the biologically inspired control of locomotion, we use an autonomous underwater vehicle (AUV) propelled by flapping foils that resemble the fore fins of a sea turtle. As shown in Figure 1, it has four flapping foils with two degrees of freedom for each. Each fin has freedom in the roll and the pitch directions, actuated by two independent electric motors. The size of the vehicle is as large as $2 \mathrm{~m} \times 0.5 \mathrm{~m} \times 0.5 \mathrm{~m}$. The top operating speed is near $2 \mathrm{~m} / \mathrm{s}$ and the flapping foils provide the whole propulsion as well as the control of the attitude and position. The detailed description of the turtle underwater vehicle can be found in [12].

\section{Top-Down CPG-BASEd Control Models}

In this section, we propose models of CPG-based control of the AUV, based on a top-down architecture. In the top-down CPG-based control architecture, there are two separate layers. One is the CPG layer composed of governing oscillators and the other is the mechanical layer composed of actuators and sensors. Sometimes the architecture is enhanced with sensory feedback from the mechanical layer to the CPG layer.

Similar models are also widely used in the bio-inspired robotics community. In our specific model, the CPG layer is based on the coupled Hopf oscillators. Notice that the artificial CPG model we use is rather a simplification of animal CPGs since we only capture properties essential to our purpose. The fins connected to motors and sensors comprise the mechanical layer.

Based on such an architecture, we propose two CPGbased controllers - one is the open-loop method without any feedback from the mechanical layer to the CPG layer while the other is a feedback approach where the coupling in the CPG layer is wholly composed of mechanical layer elements.

\section{A. Top-down Open-loop approach}

One can implement a CPG-based control law with a tracking control law that follows any oscillatory reference signal. In our top-down models, coupled Hopf oscillators form the CPG layer. A Hopf oscillator is a limit-cycle oscillator with circular symmetry on a two-dimensional plane [10], [14]. By feeding states of the oscillator to the servo system of each fin, we can coordinate the ensemble of fin motions and thus control the locomotion of the vehicle. An advantage of using coupled oscillators as the reference is, when we want to extend the system by connecting multiple modules, we have the authority for global synchronization or concurrent synchronization as discussed in [10], [14]. The model introduced in the following is a special case of [10] modified for the AUV.

Let us denote the state vector of Hopf oscillator associated with the roll control of the $i$-th fin as $\mathbf{x}_{i}$, and the one associated with the pitch control of the fin as $\mathbf{y}_{i}$. The state vectors $\mathbf{p}_{i}$ and $\mathbf{q}_{i}$ are the roll and the pitch state vectors of the $i$-th fin, respectively. Each state vector consists of angular velocity and position. A top-down CPG-based control law is proposed as

$$
\begin{aligned}
\{\dot{\mathbf{x}}\}= & \left\{\mathbf{f}_{x}\right\}(\{\mathbf{x}\})-k_{x} \mathbf{L}_{x}\{\mathbf{x}\} \\
\{\dot{\mathbf{y}}\}= & \left\{\mathbf{f}_{y}\right\}(\{\mathbf{y}\})-k_{y} \mathbf{L}_{y}\{\mathbf{y}\} \\
& +k_{x y}\left(\left[\mathbf{R}\left(\frac{\pi}{2}\right)\right]\{\mathbf{x}\}-\{\mathbf{y}\}\right) \\
\{\dot{\mathbf{p}}\}= & \left\{\mathbf{f}_{p}\right\}(\{\mathbf{p}\})+\left[\mathbf{B}_{p}\right]\{\mathbf{x}\} \\
\{\dot{\mathbf{q}}\}= & \left\{\mathbf{f}_{q}\right\}(\{\mathbf{q}\})+\left[\mathbf{B}_{q}\right]\{\mathbf{y}\},
\end{aligned}
$$

where [ ] denotes a block diagonal matrix of appropriate dimension, \{\} an aggregation of the state vectors in a column vector, and $\mathbf{R}(\phi)$ a planar rotational transformation of angle $\phi . \mathbf{L}$ is the coupling matrix in each network of oscillators distinguished by its subscript, $k$ is the scalar coupling strength for each network. The dynamics of the oscillators and the tracking controllers are given as

$$
\begin{aligned}
\mathbf{f}_{x}\left(\mathbf{x}_{i}\right) & =\mathbf{f}_{H}\left(\mathbf{x}_{i} ; \rho_{x}, \lambda_{x}\right) \\
\mathbf{f}_{y}\left(\mathbf{y}_{i}\right) & =\mathbf{f}_{H}\left(\mathbf{y}_{i} ; \rho_{y}, \lambda_{y}\right) \\
\mathbf{f}_{p}\left(\mathbf{p}_{i}\right) & =\mathbf{f}_{P D}\left(\mathbf{p}_{i} ; B_{p}, D_{p}, K_{p}, P_{p}, I_{p}\right) \\
\mathbf{f}_{q}\left(\mathbf{q}_{i}\right) & =\mathbf{f}_{P D}\left(\mathbf{q}_{i} ; B_{q}, D_{q}, K_{q}, P_{q}, I_{q}\right)
\end{aligned}
$$

with input matrices

$$
\begin{aligned}
\mathbf{B}_{p} & =\left(\begin{array}{cc}
\omega D_{p} / I_{p} & P_{p} / I_{p} \\
0 & 0
\end{array}\right) \\
\mathbf{B}_{q} & =\left(\begin{array}{cc}
\omega D_{q} / I_{q} & P_{q} / I_{q} \\
0 & 0
\end{array}\right),
\end{aligned}
$$

where

$$
\begin{aligned}
& \mathbf{f}_{H}\left(\left(u_{i}, v_{i}\right) ; \rho, \lambda\right)= \\
& \left.\qquad \begin{array}{c}
-\omega v_{i}-\lambda\left(\frac{u_{i}^{2}+v_{i}^{2}}{\rho^{2}}-1\right) u_{i} \\
\omega u_{i}-\lambda\left(\frac{u_{i}^{2}+v_{i}^{2}}{\rho^{2}}-1\right) v_{i}
\end{array}\right)
\end{aligned}
$$

and $\mathbf{f}_{P D}\left(\left(u_{i}, v_{i}\right) ; B, K, D, P, I\right)$ is a general PD control law; $\omega$ is the common frequency for roll and pitch; $\rho$ the limit cycle radius; $\lambda$ the convergence rate to the limit cycle; $B$ 
the damping coefficient; $D$ the differential gain; $K$ the spring constant; $P$ the proportional gain; and $I$ the moment of inertia.

Notice that (1) and (2) represent a network of coupled oscillators as a special case of [10] while (3) and (4) represent PD controlled mass-spring-damper systems. Hence, the first two consist the CPG layer and the latter two the mechanical layer.

Using partial contraction analysis as in [10], [14], [15], one can find a lower limit of $k>0$ that ensures the exponential synchronization of the oscillators in the CPG layer. Once synchronized, the diffusively coupled terms (e.g., $q_{1}-q_{2}$ ) vanish and thus each oscillator behaves as if it were uncoupled to exhibit its intrinsic limit-cycle behavior. The sinusoidal output $v_{i}$ of $\mathbf{f}_{H}$ is used as a reference input for the position tracking system of the roll and the pitch controllers. One condition on $\mathbf{L}$ is that it should represent a connected network [14].

We can also set an arbitrary phase bias between oscillators in the CPG layer by adjusting $\mathbf{L}$. The synchronization with phase difference is proved in [10], [14]. For example, one can set $\mathbf{L}_{x}$ as follows for two-way ring couplings:

$$
\mathbf{L}_{x}=\left(\begin{array}{cccc}
2 \mathbf{I} & -\mathbf{R}\left(\phi_{12}\right) & -\mathbf{R}\left(\phi_{13}\right) & \mathbf{0} \\
-\mathbf{R}\left(\phi_{21}\right) & 2 \mathbf{I} & \mathbf{0} & -\mathbf{R}\left(\phi_{24}\right) \\
-\mathbf{R}\left(\phi_{31}\right) & \mathbf{0} & 2 \mathbf{I} & -\mathbf{R}\left(\phi_{34}\right) \\
\mathbf{0} & -\mathbf{R}\left(\phi_{42}\right) & -\mathbf{R}\left(\phi_{43}\right) & 2 \mathbf{I}
\end{array}\right)
$$

with $\phi_{i j}=-\phi_{j i}$. By setting $\phi_{i j}=0$ for all $i$ and $j$, one can establish the in-phase synchronization for roll motions. Setting $\phi_{i j}=0$ for $i+j=0 \bmod 2$ and $\phi_{i j}=\pi$ for $i+j=1 \bmod 2$ yields the bound gait where the fins are synchronized ipsilaterally out of phase and contralaterally in phase. Exponential synchronization implies that the change of phase bias at any moment yields exponentially fast convergence to a new pattern.

Essentially, in the top-down open-loop approach, the coupled oscillators generate coordinated reference signals for the fin actuators. The amplitude and the frequency can be modulated by commanding a small number of parameters such as $\rho$ and $\omega$, thereby reducing the control space.

\section{B. Top-down CPG with Feedback Coupling}

One drawback of the open-loop CPG is that the synchronization occurs only in the CPG level and the synchronization of fin motions depends on the performance of the position tracking controller. One can easily consider a scenario where the fins are not ideally identical or the position tracking systems have slightly different performance among fins. As a result, the fins remain slightly unsynchronized while the reference signals are synchronized. To overcome the limit of the top-down open-loop CPG controller, the couplings in CPG are modified to accommodate the state of the fins as in

$$
\begin{aligned}
\{\dot{\mathbf{x}}\}= & \left\{\mathbf{f}_{x}\right\}(\{\mathbf{x}\})-k_{x} \mathbf{L}_{x}\{\mathbf{p}\} \\
\{\dot{\mathbf{y}}\}= & \left\{\mathbf{f}_{y}\right\}(\{\mathbf{y}\})-k_{y} \mathbf{L}_{y}\{\mathbf{q}\} \\
& +k_{x y}\left(\left[\mathbf{R}\left(\frac{\pi}{2}\right)\right]\{\mathbf{p}\}-\{\mathbf{q}\}\right)
\end{aligned}
$$

$$
\begin{aligned}
\{\dot{\mathbf{p}}\} & =\left\{\mathbf{f}_{p}\right\}(\{\mathbf{p}\})+\left[\mathbf{B}_{p}\right]\{\mathbf{x}\} \\
\{\dot{\mathbf{q}}\} & =\left\{\mathbf{f}_{q}\right\}(\{\mathbf{q}\})+\left[\mathbf{B}_{q}\right]\{\mathbf{y}\} .
\end{aligned}
$$

By applying differential coordinate transformations in (3) and (4), one can see that the position tracking system is semicontracting in the absence of the input. Hence, the solutions forget the initial conditions asymptotically, and after some transient time they oscillate at the frequency of the input signals. The amplitude and the phase lag can be computed given the input frequency. By ignoring the transient behavior, we can reduce the preceding model in (13-16) as

$$
\begin{array}{r}
\{\dot{\mathbf{x}}\}=\left\{\mathbf{f}_{x}\right\}(\{\mathbf{x}\})-k_{x} \mathbf{L}_{x}\left\{A_{p} \mathbf{R}\left(\phi_{x p}\right) \mathbf{x}\right\} \\
\{\dot{\mathbf{y}}\}=\left\{\mathbf{f}_{y}\right\}(\{\mathbf{y}\})-k_{y} \mathbf{L}_{y}\left\{A_{q} \mathbf{R}\left(\phi_{y q}\right) \mathbf{y}\right\} \\
+k_{x y}\left(\left[\mathbf{R}\left(\frac{\pi}{2}\right)\right]\left\{A_{p} \mathbf{R}\left(\phi_{x p}\right) \mathbf{x}\right\}-\left\{A_{q} \mathbf{R}\left(\phi_{y q}\right) \mathbf{y}\right\}\right),
\end{array}
$$

where $A_{p}=A_{p}(\omega)$ (or $A_{q}=A_{q}(\omega)$ ) is the amplitude as a function of the eigenfrequency $\omega$, and $\phi_{x p}=\phi_{x p}(\omega)$ (or $\phi_{y q}=\phi_{y q}(\omega)$ ) is the phase lag from $\mathbf{x}$ to $\mathbf{p}$ (or $\mathbf{y}$ to $\mathbf{q}$, respectively), also a function of $\omega$.

The performance of the proposed model depends on the dynamics of $\mathbf{p}$ and $\mathbf{q}$. If the dynamics is more complex than a mass-spring-damper as we assumed, the direct coupling e.g., between $\mathbf{x}$ and $\mathbf{p}$ could disturb the CPG layer. When $\phi_{x p}$ is larger than $\pi$, the system bifurcates to anti-synchronization, where the coupling acts like repulsion instead of attraction.

\section{Single LAYER ARChitecture}

While the top-down approach can serve as a good motionplanning method from its simplicity and modularity, the synchronization via coupling is limited only to the CPG oscillators. Actual synchronization of the fins are affected by how identical the dynamics of the fins are. To extend the synchronization of the coupled oscillators beyond the layer of the CPG oscillators to the actual fins, we propose a control law that induces self-sustained oscillation in the fin motions and also enforces direct synchronization of the oscillations.

In this section, we let oscillations emerge from the mechanical layers by feeding a nonlinear function of velocity back to the motor torque. The result is comparable to the neuro-mechanical oscillation discussed in [13] in that the oscillation is induced by coupling non-oscillatory elements. As a result, the CPG layer is eliminated from the top-down architecture to form a single-layer architecture. On top of the oscillating fins, coupling input for synchronization is applied within the mechanical layer. In summary, we can synchronize the oscillation of fins without using a reference oscillator.

One can model the servo-actuated fins by using EulerLagrange equations. The aim here is to apply nonlinear state feedback to construct a limit cycle oscillator. Consider the following second-order coupled roll-pitch actuator dynamics

$$
\mathbf{M}(\mathbf{p}, \mathbf{q})+\mathbf{C}(\mathbf{p}, \mathbf{q}, \dot{\mathbf{p}}, \dot{\mathbf{q}})+\mathbf{K}(\mathbf{p}, \mathbf{q})=\left(\tau_{\text {roll }}, \tau_{\text {pitch }}\right)^{T}
$$

In order to focus on verifying the feasibility of the proposed closed-loop CPG method, let us assume that the coupling 
between $\mathbf{p}$ and $\mathbf{q}$ is relatively small. Then, each decoupled dynamics can be represented by

$$
I \ddot{x}+B(\dot{x}) \dot{x}+K x=\tau,
$$

where $I$ is the moment of inertia, $B$ the nonlinear damping term as a function of $\dot{x}, K$ the spring constant, and $\tau$ the input torque. $x$ represents angular displacement, and $\dot{x}$ angular velocity.

The nonlinear damping of the fin in the fluid can be modelled as

$$
B(\dot{x})=\beta_{0}+\beta_{1}|\dot{x}|,
$$

where $\beta_{0}, \beta_{1}>0$. The term led by $\beta_{1}$ is justified by the experimental observation [12], [16], where the lift force of a single fin oscillating in the fluid is in phase with the angular velocity and its magnitude is proportional to the angular velocity squared.

\section{A. Velocity feedback}

Let us design a torque control law as

$$
\tau=-P x+\gamma_{0} \dot{x}-\gamma_{1}|\dot{x}| \dot{x}+I s(t),
$$

where $s(t)$ is a synchronizing input to be discussed further below. The closed-loop dynamics of (20) and (22) is

$$
I \ddot{x}+\left(\beta_{0}-\gamma_{0}+\beta_{1}|\dot{x}|+\gamma_{1}|\dot{x}|\right) \dot{x}+(K+P) x=I s(t) .
$$

After dividing the equation by $I$ and setting $\omega_{0}=\sqrt{\frac{K+P}{I}}$ and $C(\dot{x})=\frac{\beta_{0}-\gamma_{0}+\beta_{1}|\dot{x}|+\gamma_{1}|\dot{x}|}{I}$, we have

$$
\ddot{x}+C(\dot{x}) \dot{x}+\omega_{0}^{2} x=s(t) .
$$

By choosing $\gamma_{0}$ and $\gamma_{1}$ to satisfy $\beta_{0}-\gamma_{0}<0$ and $\beta_{1}+$ $\gamma_{1}>0$, the system shows limit cycle behavior. If we denote $\left(\gamma_{0}-\beta_{0}\right) / I=\sigma_{0}$ and $\left(\beta_{1}+\gamma_{1}\right) / I=\sigma_{1}$, then

$$
\ddot{x}+\left(\sigma_{1}|\dot{x}|-\sigma_{0}\right) \dot{x}+\omega_{0}^{2} x=s(t) .
$$

The resulting dynamics shows limit cycle property. Different feedback controllers yield different types of limit cycle oscillators and we may also use them to control the AUV.

We can deliberately select the values $\sigma_{1}, \sigma_{0}$, and $\omega_{0}$ for the feedback controller to shape the limit cycle and its frequency. Depending on the scale of the nonlinearity in the damping term, we can compute its amplitude and frequency as follows.

\section{B. Weak Nonlinearity}

For a weakly nonlinear case, i.e., for $\sigma_{1} \ll 1$ and $\sigma_{0} \ll 1$, the phase portrait is close to a circle. We can apply singular perturbation theory for two-time scales (see [17], [18]) to estimate the amplitude and frequency of the oscillation when it is uncoupled $(s(t)=0)$.

The small parameters $\sigma_{0}, \sigma_{1} \ll 1$ can be written using $\epsilon \ll$ 1 and $0<B_{0}, B_{1} \sim \mathcal{O}(1)$ as in

$$
\ddot{x}+\epsilon\left(B_{1}|\dot{x}|-B_{0}\right) \dot{x}+w_{0}^{2} x=0 .
$$

The amplitude of oscillation is an important parameter to generate proper swimming motion. We can assume a sinusoidal solution with amplitude $r$. The amplitude $r$ is found to have "slow" dynamics as

$$
r^{\prime}=-\frac{1}{2} w_{0}^{-1} B_{0} r+B_{1} r|r| \frac{4}{3 \pi},
$$

where $r^{\prime}$ is the time derivative of $r$ with respect to the "slow" time. Its stable equilibrium is found at $r_{\infty}=\frac{3 \pi}{8 w_{0}} \frac{B_{0}}{B_{1}}$. The frequency of motion is not modulated during the swimming. However, we need to set the frequency at a certain range to ensure agility of the vehicle. It is dealt with the perturbation theory as well. The result is found as $\omega=\omega_{0}+\mathcal{O}\left(\epsilon^{2}\right)$.

\section{Strong Nonlinearity}

In the strong nonlinearity, represented by $\sigma_{0}, \sigma_{1} \gg 1$, the phase portrait looks distorted compared to that of weakly nonlinear oscillator. We replace $\epsilon$ in (26) with $\mu \gg 1$ to have

$$
\ddot{x}+\mu\left(B_{1}|\dot{x}|-B_{0}\right) \dot{x}+\omega_{0}^{2} x=0 .
$$

It is often called a relaxation oscillator. The name follows from the fact that the cycle of oscillation composed of slow build-up and fast relaxation. After some work on the analysis on the phase portrait, one can find an approximate period and amplitude of the solution $x(t)$ as follows. The period is found as

$$
T=\frac{2 \mu}{\omega_{0}^{2}}\left(B_{0} \ln \frac{B_{0}}{B_{0}+\sqrt{B_{0}\left(B_{0}+\omega_{0}\right)}}+\sqrt{B_{0}\left(B_{0}+\omega_{0}\right)}\right)
$$

and the amplitude of oscillation can also be approximated by considering its nullclines as $r_{\infty}=\frac{\mu B_{0}}{4 \omega_{0} B_{1}}$.

\section{Bias}

The center of oscillation has been at the origin through the discussion so far, which can be extended to a biased oscillation centered at $x=x_{0}$. To implement a bias $x_{0}$ in $x$, the feedback input must be modified as

$$
\tau=-P x+\gamma_{0} \dot{x}-\gamma_{1}|\dot{x}| \dot{x}+I s(t)+(K+P) x_{0}
$$

and the closed loop dynamics becomes

$$
\ddot{x}+C(\dot{x}) \dot{x}+\omega_{0}^{2}\left(x-x_{0}\right)=s(t) .
$$

One can define a new variable such as $x^{\prime}=x-x_{0}$ to apply the results above.

\section{E. Integration with Underwater Vehicle Control System}

For each cycle of the oscillation, the main CPU determines the oscillation parameters such as the amplitude, frequency, and bias by comparing the current attitude and the desired attitude. Such updates of the oscillation parameters are performed at a much slower rate than the sampling rate of the feedback controller that governs the oscillatory motion of fins. The oscillation parameters commanded by the main CPU need to be mapped to the parameters for the feedback controller. Although we derived all the equations regarding how the parameters of the feedback controller determines the oscillator 
parameters, there are some unknown constants that present the physical property of the system. It is also possible that there are some dynamics that are not accounted for in (20). Hence, for the successful implementation of the proposed CPG-based controller, we chose to determine the relation of controller parameters versus oscillation parameters through experimental tests and the curve fitting method. To determine the order of the curve, the equations derived above are helpful.

\section{F. Coupling Input for Synchronization}

The synchronizing input for $i$-th system $s_{i}(t)$ can be designed in various ways. If we assume the dynamics of the oscillators are identical, then we can use

$$
s_{i}(t)=\kappa\left(y_{j}-y_{i}\right),
$$

where $y_{i}=\dot{x}_{i} / \omega_{0}$. Partial contraction analysis, similar to that in [15], can show that they will synchronize asymptotically for strong enough coupling gain $\kappa>0$. Furthermore, to force the oscillators to synchronize with a phase difference of $\phi$, one can use

$$
s_{i}(t)=\kappa\left(\sin (\phi) x_{j}+\cos (\phi) y_{j}-y_{i}\right) .
$$

Notice that the phase $\phi_{i}$ of the state $\left(x_{i}, y_{i}\right)$ can be defined in terms of $x_{i}$ and $y_{i}$ as $\phi_{i}=\tan ^{-1} \frac{y_{i}}{x_{i}}$.

As for the synchronization of the roll oscillator and the pitch oscillator, it is often desired that their amplitudes remain independent. In that case, one can simply modify the coupling by scaling with the estimate amplitudes $r_{i}$ and $r_{j}$ of the oscillations as in

$$
s_{i}(t)=\kappa\left(\frac{r_{i}}{r_{j}} y_{j}-y_{i}\right) .
$$

To present the coupling input $s_{i}(t)$ for synchronization in a brief form, let us define $\mathbf{x}_{i}=\left(x_{i}, y_{i}\right)^{\mathrm{T}}, \mathbf{R}(\phi) \in \mathcal{S O}(2)$ to be a planar rotational transformation of angle $\phi$, and $\mathbf{S}=\left(\begin{array}{ll}0 & 1\end{array}\right)$. Then, after integrating the discussion above on $s_{i}(t)$, we can propose the coupling input for synchronization to the fin $i$ as

$$
s_{i}(t)=\kappa \mathbf{S}\left(\frac{r_{i}}{r_{j}} \mathbf{R}(\phi) \mathbf{x}_{j}-\mathbf{x}_{i}\right) .
$$

\section{G. Synchronization Analysis for One-way Ring Network}

Using partial contraction theory and its extended theorems in [15], one can analyze the stability of synchronization.

1) Special Case with $\phi=0, \pi$ : The analysis for asymptotical synchronization with phase difference of $\phi=0$ or $\pi$ is found in [15] for van der Pol oscillators. Below, we derive the same conclusion using the method of projected jacobian introduced in [14].

Let us start with the synchronization of roll oscillations with $\phi=0$. The result can be easily extended for $\phi=\pi$. The angular position and the angular velocity of the roll motions are represented by a vector $\mathbf{x}_{i}$ for fin $i$. For $\phi=0$, we have $\mathbf{R}(0)=\mathbf{I}$ and the dynamics of the coupled oscillators can be written as

$$
\dot{\mathbf{x}}_{i}=\left(\begin{array}{cc}
0 & \omega_{0} \\
-\omega_{0} & \sigma_{0}-\sigma_{1} \omega_{0}\left|y_{i}\right|
\end{array}\right) \mathbf{x}_{i}+k \mathbf{K}\left(\mathbf{x}_{j}-\mathbf{x}_{i}\right),
$$

where $k=\kappa \omega^{-1}$ and

$$
\mathbf{K}=\left(\begin{array}{ll}
0 & 0 \\
0 & 1
\end{array}\right)
$$

with $j=i+1$ (modulo 4) for the one-way ring network.

The subspace for synchronization is $\mathcal{M}=\left\{\mathbf{x}_{1}=\mathrm{x}_{2}=\right.$ $\left.\mathbf{x}_{3}=\mathbf{x}_{4}\right\}$, which is verified to be flow-invariant under (36). According to [14], if the jacobian of the projected dynamics on $\mathcal{M}^{\perp}$ has negative definite symmetric part, then the system is contracting toward $\mathcal{M}$, i.e., synchronizing.

The jacobian $\mathbf{J}_{i}$ of uncoupled dynamics of an oscillator is

$$
\mathbf{J}_{i}=\left(\begin{array}{cc}
0 & \omega_{0} \\
-\omega_{0} & \sigma_{0}-2 \sigma_{1} \omega_{0}\left|y_{i}\right|
\end{array}\right)
$$

and its symmetrical part $\mathbf{J}_{i s}$ is

$$
\mathbf{J}_{i s}=\left(\begin{array}{cc}
0 & 0 \\
0 & \sigma_{0}-2 \sigma_{1} \omega_{0}\left|y_{i}\right|
\end{array}\right) .
$$

Define

$$
\mathbf{L}=\left(\begin{array}{cccc}
\mathbf{K} & -\mathbf{K} & \mathbf{0} & \mathbf{0} \\
\mathbf{0} & \mathbf{K} & -\mathbf{K} & \mathbf{0} \\
\mathbf{0} & \mathbf{0} & \mathbf{K} & -\mathbf{K} \\
-\mathbf{K} & \mathbf{0} & \mathbf{0} & \mathbf{K}
\end{array}\right)
$$

and its symmetric part as $\mathbf{L}_{s}$. Now the jacobian of the coupled oscillator has its symmetric part as

$$
\mathbf{J}_{s}=\left[\mathbf{J}_{i s}\right]-k \mathbf{L}_{s},
$$

where [ ] denotes a block diagonal form. An orthogonal projection $\mathbf{V}$ to $\mathcal{M}^{\perp}$ can be found as [19]

$$
\mathbf{V}=\frac{1}{2}\left(\begin{array}{cccc}
\mathbf{I} & -\mathbf{I} & \mathbf{I} & -\mathbf{I} \\
\mathbf{0} & -\sqrt{2} \mathbf{I} & \mathbf{0} & \sqrt{2} \mathbf{I} \\
-\sqrt{2} \mathbf{I} & \mathbf{0} & \sqrt{2} \mathbf{I} & \mathbf{0}
\end{array}\right)
$$

using the eigenvectors of $\mathbf{L}_{s}$.

From [14], if the projected jacobian $\mathbf{P}_{s}=\mathbf{V} \mathbf{J}_{s} \mathbf{V}^{\mathrm{T}}$ is uniformly negative definite, then $\mathbf{V}\{\mathbf{x}\}$ converges to $\mathbf{0}$, which is equivalent to $\{\mathbf{x}\}$ converging to $\mathcal{M}$.

Ruling out the zero columns and rows from $\mathbf{P}_{s}$ yields

$$
\overline{\mathbf{P}}_{s}=\overline{\mathbf{V}\left[\mathbf{J}_{i s}\right] \mathbf{V}^{\mathrm{T}}}-k\left(\begin{array}{ccc}
2 & 0 & 0 \\
0 & 1 & 0 \\
0 & 0 & 1
\end{array}\right),
$$

where $\overline{()}$ denotes the remaining part after removing the zero columns and rows. The eigenvalues of $\overline{\mathbf{V L}_{s} \mathbf{V}^{\mathrm{T}}}$ is $(2,1,1)$ and

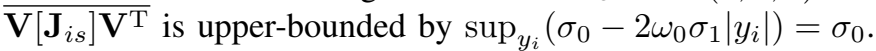

Hence, a sufficient condition for $\overline{\mathbf{P}_{s}}$ to be negative definite is $k>\sigma_{0}$. Since the removed columns and rows correspond to positions $x_{i}$, the negative definiteness of $\overline{\mathbf{P}}_{s}$ implies that $\mathbf{P}_{s}$ negative semi-definite (semi-contracting). By Barbalat's lemma, it is straightforward to show that the velocities $\dot{x}_{i}$ synchronize asymptotically from any initial condition. Once the oscillators synchronize their velocities, i.e., $\dot{x}_{i}-\dot{x}_{j} \rightarrow 0$, the coupling inputs $s_{i}$ vanish, resulting in a stable limit cycle. Since $\delta\{\mathbf{x}\}^{T} \delta\{\mathbf{x}\}$ tends to a lower limit asymptotically, its higher-order Taylor expansion, similar to [15], indicates that the angular positions on the resulting limit cycle synchronize as well, i.e., $x_{i}-x_{j} \rightarrow 0$. 
2) General Case with Arbitrary Phase Bias: The following is a general form in the sense that it accommodates an arbitrary phase bias $\phi$ in the coupling as well as scaling for amplitude difference as $r_{i} / r_{j}$.

$$
\begin{aligned}
\dot{\mathbf{x}}_{i}= & \left(\begin{array}{cc}
0 & \omega_{0} \\
-\omega_{0} & \sigma_{0}-\sigma_{1} \omega_{0}\left|y_{i}\right|
\end{array}\right) \mathbf{x}_{i} \\
& +k \mathbf{K}\left(\frac{r_{i}}{r_{j}} \mathbf{R}(\phi) \mathbf{x}_{j}-\mathbf{x}_{i}\right),
\end{aligned}
$$

where $\mathbf{K}$ is defined in (37) and set $j=i+1$ with modulo 4 for a one-way ring network. We use shorthand notation $\mathbf{T}_{i-1}=$ $\frac{r_{i}}{r_{i+1}} \mathbf{R}((i-1) \phi)$. Also, we assume that the phase bias $\phi$ is the same for each oscillator. For the same one-way ring topology represented by (40), we present the proof of the synchronization of $\mathbf{x}_{i}$ to $\mathcal{M}=\left\{\mathbf{T}_{i} \mathbf{x}_{i+1}=\mathbf{T}_{i-1} \mathbf{x}_{i}, \forall i \bmod 4\right\}$. Hence, the flow-invariant set $\mathcal{M}$ contains phase-shifted variables such as $\mathbf{x}_{1}=\mathbf{T}_{1} \mathbf{x}_{2}=\mathbf{T}_{2} \mathbf{x}_{3}=\mathbf{T}_{3} \mathbf{x}_{4}$. Therefore, $\prod_{i}^{n} \mathbf{T}_{i-1}=\mathbf{I}$ is required as a constraint.

For simplicity, let us assume $r_{j}=r_{i}$. (For $r_{j} \neq r_{i}$, one can use the coordinate transformation introduced in [10]). If we define $\mathbf{z}_{i}=\mathbf{R}((i-1) \phi) \mathbf{x}_{i}=\mathbf{T}_{i-1} \mathbf{x}_{i}$, then

$$
\mathbf{x}_{i+1}=\mathbf{R}(-\phi) \mathbf{x}_{i} \Leftrightarrow \mathbf{z}_{i+1}=\mathbf{z}_{i} .
$$

The virtual system dynamics for $\delta \mathbf{z}_{i}$ can be obtained by leftmultiplying (44) with $\mathbf{T}_{i-1}$ :

$$
\delta \dot{\mathbf{z}}_{i}=\left(\mathbf{T}_{i-1} \mathbf{J}_{i} \mathbf{T}_{i-1}^{T}\right) \delta \mathbf{z}_{i}+k \mathbf{T}_{i-1} \mathbf{K} \mathbf{T}_{i-1}^{T}\left(\mathbf{z}_{i+1}-\mathbf{z}_{i}\right)
$$

Let us introduce the shorthand notation $\mathbf{K}_{T i}=\mathbf{T}_{i-1} \mathbf{K T}_{i-1}^{T}$. The jacobian $\mathbf{J}_{z}$ of the congregated system in the space of $\mathbf{z}$ is

$$
\mathbf{J}_{z}=\left[\mathbf{T}_{i-1} \mathbf{J}_{\mathbf{i}} \mathbf{T}_{i-1}^{T}\right]-k \mathbf{L}_{z},
$$

where [ ] is a notation for block diagonal matrix, $\mathbf{J}_{\mathbf{i}}$ is defined from (38), and

$$
\mathbf{L}_{z}=\left(\begin{array}{cccc}
\mathbf{K}_{T 1} & -\mathbf{K}_{T 1} & \mathbf{0} & \mathbf{0} \\
\mathbf{0} & \mathbf{K}_{T 2} & -\mathbf{K}_{T 2} & \mathbf{0} \\
\mathbf{0} & \mathbf{0} & \mathbf{K}_{T 3} & -\mathbf{K}_{T 3} \\
-\mathbf{K}_{T 4} & \mathbf{0} & \mathbf{0} & \mathbf{K}_{T 4}
\end{array}\right) .
$$

Notice that the eigenvalues of the symmetric part of $\mathbf{T}_{i-1} \mathbf{J}_{i} \mathbf{T}_{i-1}^{T}$ are equal to those of the symmetric part of $\mathbf{J}_{i}$. The eigenvalues of the symmetric part of $\mathbf{L}_{z}$ also agree with the Laplacian $\mathbf{L}$ in (40). Hence, the proof in the previous section still holds. As a result, we can conclude that the oscillators asymptotically converge to the flow-invariant manifold $\mathcal{M}$ of phase synchronization for any $k>\sigma_{0}$. This result holds generally for an arbitrarily large number of oscillators with phase shift $\phi$.

If we assign the first oscillator to the fore-left fin, the second to the hind-left fin, the third to the fore-right fin, and the fourth to the hind-right fin, then using the one-way ring structure allows us to implement such gaits [20] as walk, bound, and pronk by setting $\phi=\frac{\pi}{2}, \pi$ and 0 , respectively.

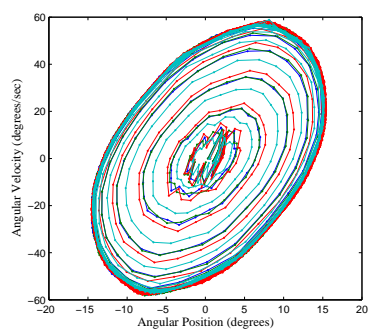

(a)

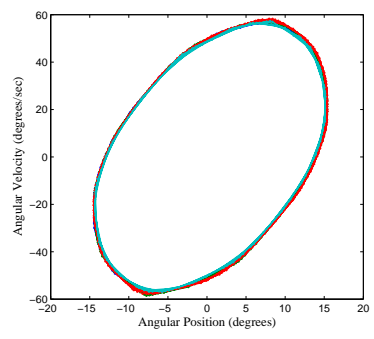

(b)
Fig. 2. The states of the four fins are plotted for angles versus filtered angular rate with respect to the roll axis. The circular trajectory and slow convergence to the limit cycle are the characteristics of weak nonlinearity. (a) The oscillation grows from the origin. (b) The trajectories after 25 seconds are plotted to illustrate limit cycle clearly.

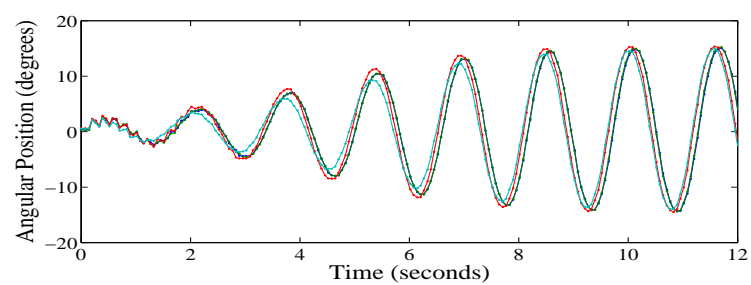

Fig. 3. Roll positions of the four fins are plotted when the synchronization is not applied. The phase differences persist.

\section{H. Network of the oscillators for AUV Fins}

The following is the principles that we put on the design of the CPG network for AUV fins.

1) The coupling from a roll oscillator to a pitch oscillator of the same fin is one-way and one-to-one with a 90degree phase shift.

2) Roll oscillators are coupled with each other and the phase bias between oscillators can be arbitrarily chosen.

3) Pitch oscillators are not connected to each other.

Once the roll oscillators synchronize themselves, we can show that the pitch oscillators also synchronize with the associated roll oscillators in a leader-follower fashion. We refer the readers to [14], [15], [21] for the detailed proof of the leaderfollower synchronization using contraction theory. Although the pitch and roll oscillators have different dynamics and different frequencies, after simplifying the model as phase oscillators, one can show that they synchronize to oscillate at a common frequency with some constant phase delay that depends on the coupling strength and the difference between the intrinsic frequencies [22].

\section{EXPERIMENTS USING THE SINGLE-LAYER APPROACH}

We experimentally validated the feasibility of the proposed single-layer CPG-based control for synchronized fin motions with the turtle AUV. After observing that the oscillation actually occurs by using the velocity feedback and that the roll and pitch motions of a fin show stable limit-cycle behaviors, the coupling among the oscillators was activated. Figure 2(a) shows the phase portrait of the oscillation. The $x$-axis 


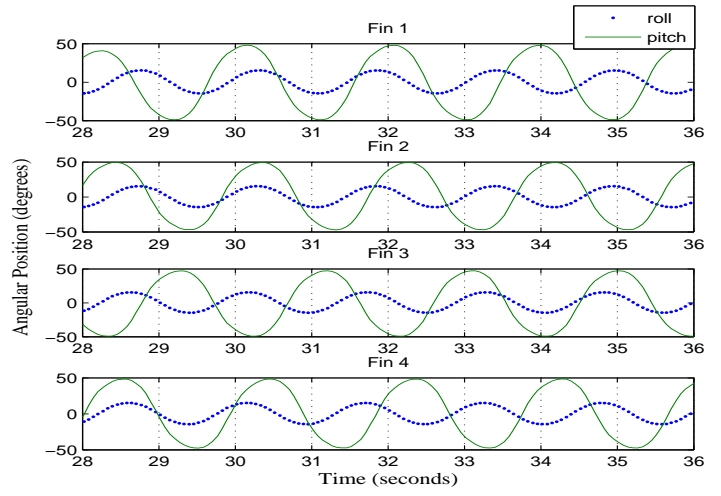

Fig. 4. Unsynchronized behaviors of roll and pitch motions of the four fins when the coupling for synchronization is not applied.

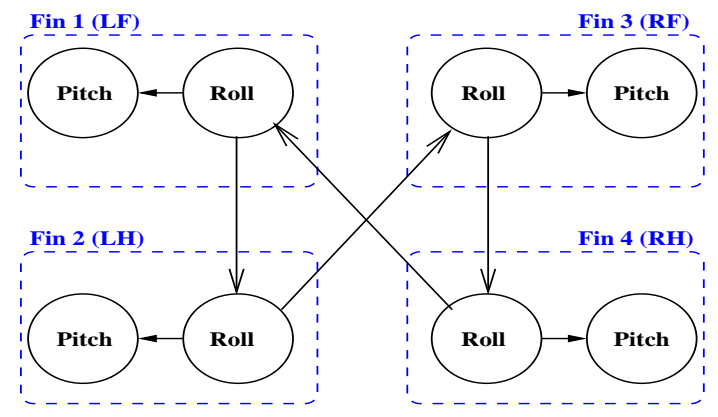

Fig. 5. One-way ring architecture was used to achieve the synchronization of roll motions among fins. Pitch motion synchronizes to its corresponding roll motion.

represents the angular position and the $y$-axis the angular velocity. The initial condition is near the origin, and the spiral trajectories grow outward to converge to the limit cycle. Figure 2(b) shows the trajectories after 25 seconds to clearly show the resulting limit cycle. The time series of the roll positions are plotted in Figure V, where one can see the motions are not fully synchronized without the couplings between them. The roll motions and pitch motions are plotted together without any couplings to indicate their unsynchronized behaviors (see Figure 4). Notice that their frequencies are also different.

To achieve the synchronization of the fin motions, we applied one-way diffusive ring couplings for the roll controllers of the turtle AUV. For the pitch controllers, we added one-way diffusive coupling with a 90-degree phase lag from the corresponding roll controllers. The coupling scheme is illustrated in Figure 5.

Figure 6 shows the synchronized roll and pitch motions of all four fins. We commanded the bound gait after 25 seconds by applying a phase bias of $\pi$ in the one-way ring network of roll motions. In Figure 7(a), the pattern starts to shift from the pronk gait (4-fin in-phase synchronization) to the bound gait (the fore fins are out of phase from the hind fins). Since the transition occurred slowly, Figure 7(b) shows the correct bound gait about 30 seconds after the phase bias is changed to $\pi$.

Figure 8 demonstrates the property of robustness of the

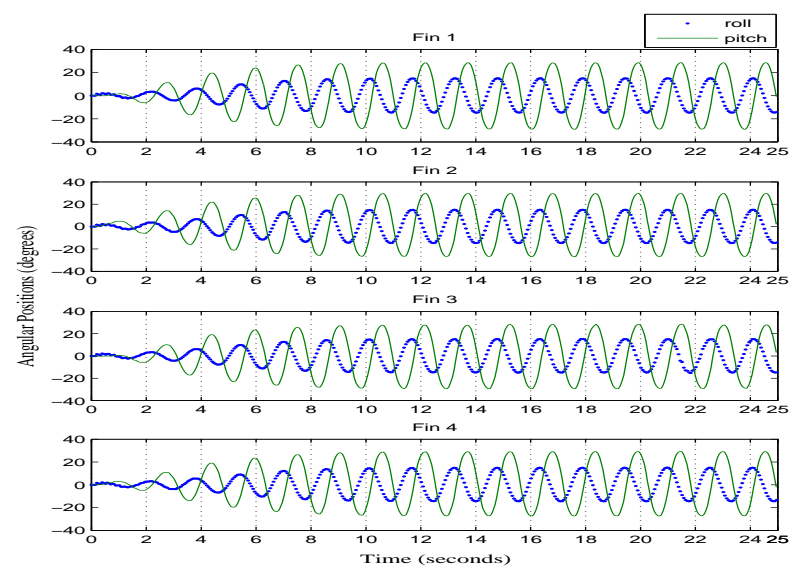

Fig. 6. Synchronized behaviors of roll and pitch motions of the four fins: all the roll motions are synchronized among themselves and the pitch motions are phase locked to the roll motions with 90 -degree phase lag

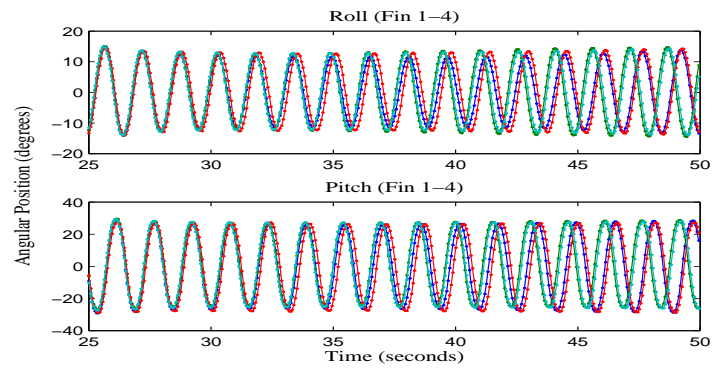

(a)

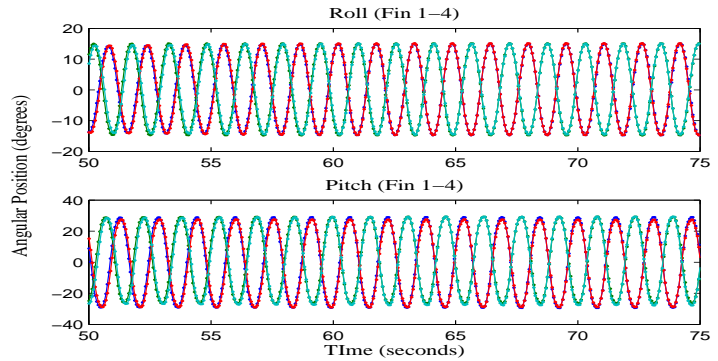

(b)

Fig. 7. Gait transition starts at $\mathrm{t}=25 \mathrm{~s}$. (a)The synchronization starts bifurcation. (b)The new pattern "bound" gait settles around $t=55 \mathrm{~s}$.
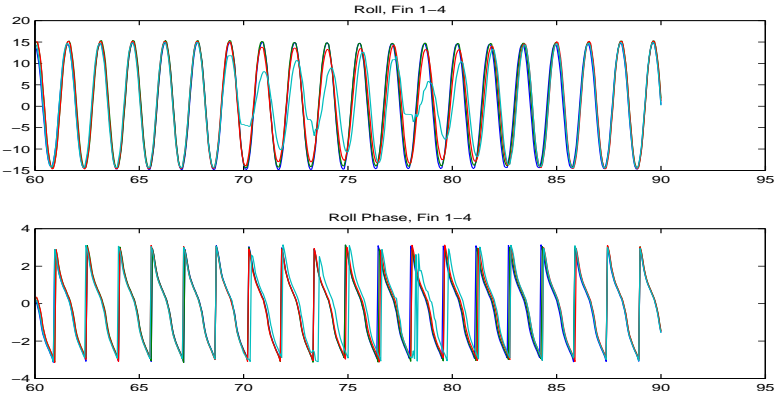

Fig. 8. A fin was disturbed and then recovered back to its synchronized rolling motion. 


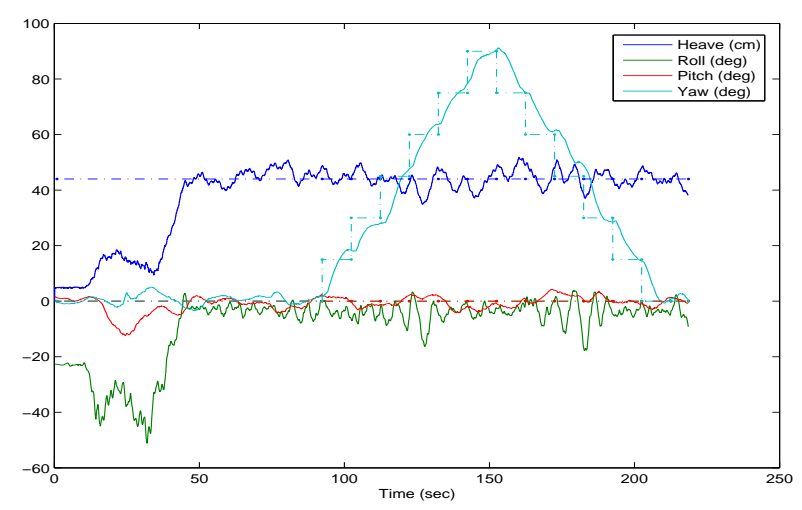

Fig. 9. Underwater mission to follow the reference yaw, pitch, roll angles and heave (depth) was performed using the proposed CPG-based controller.

proposed controller. The rolling motion was disturbed by human intervention and the motion recovers to its limit cycle while all the four fins recover to synchronization.

Finally, to demonstrate the feasibility of the proposed CPGbased controller for underwater missions, the attitude control of the vehicle was tested in a water tank in the MIT Tow Tank Lab. The vehicle is neutrally buoyant and the center of the gravity is located higher than the center of buoyancy, hence the vehicle cannot maintain its attitude without proper controller. Figure 9 shows results of underwater experimentation, where the turtle robot follows the reference heave, pitch, roll, and yaw angles by synchronized oscillatory motions of the foil fins.

\section{CONCLUSiON}

We first introduced several conventional top-down CPGbased control strategies for biologically inspired robot locomotion. In order to improve the synchronization performance of the actual fin states, we developed and experimentally validated the new CPG-based approach for a biomimetic AUV. The proposed method can be summarized as follows. By applying a nonlinear velocity feedback, we rendered each fin actuator to exhibit stable limit cycle dynamics. Further, the coupling inputs were added to synchronize multiple fins from any initial conditions. By adjusting the phase bias parameters in the coupling gains, we could also implement gait transitions. The proposed approach was experimentally validated using the turtle-like underwater vehicle. The proposed CPG-based method successfully controlled the attitude and altitude of the underwater vehicle by synchronizing the actuator foil fins in the presence of external disturbances.

\section{ACKNOWLEDGMENT}

The authors thank Prof. Michael Triantafyllou for letting them borrow the robotic turtle developed in his laboratory, as well as Stephen Licht for extensive help with the implementation and inspiring discussions on the experiments at the MIT Tow Tank. The first and second authors were partially supported by the Air Force Office of Scientific Research, and thank Prof. James Oliver at the Virtual Reality Application Center, Iowa State University.

\section{REFERENCES}

[1] S. Hirose, Biologically inspired robots : snake-like locomotors and manipulators. Oxford University Press, Oxford, New York, 1993.

[2] M. Triantafyllou and G. Triantafyllou, "An efficient swimming machine," Scientific American, pp. 64-70, March 1995.

[3] A. Crespi and A. Ijspeert, "Amphibot II: an amphibious snake robot that crawls and swims using a central pattern generator," in Proceedings of the 9th International Conference on Climbing and Walking Robots (CLAWAR 2006), Brussels, Belgium, September 2006, pp. 19-27.

[4] G. S. Stent, W. B. Kristan, Jr., W. O. Friesen, C. A. Ort, M. Poon, and R. L. Calabrese, "Neuronal generation of the leech swimming movement," Science, New Series, vol. 200, no. 4348, pp. 1348-1357, June 1978.

[5] N. Krouchev, J. F. Kalaska, and T. Drew, "Sequential activation of muscle synergies during locomotion in the intact cat as revealed by cluster analysis and direct decomposition," Journal of Neurophysiology, vol. 96, pp. 1991-2010, October 2006.

[6] I. A. Rybak, N. A. Shevtsova, M. Lafreniere-Roula, and D. A. McCrea, "Modelling spinal circuitry involved in locomotor pattern generation: insights from deletions during fictive locomotion," Journal of Physiology, vol. 577, no. 2, pp. 617-639, December 2006.

[7] G. Taga, "A model of the neuro-musculo-skeletal system for anticipatory adjustment of human locomotion during obstacle avoidance," Biological Cybernetics, vol. 78, no. 1, pp. 9-17, 1998.

[8] J. Morimoto, G. Endo, J. Nakanishi, S.-H. Hyon, G. Cheng, D. Bentivegna, and C. G. Atkeson, "Modulation of simple sinusoidal patterns by a coupled oscillator model for biped walking," in Proceedings of the 2006 IEEE International Conference on Robotics and Automation, May 2006, pp. 1579-1584.

[9] A. Ijspeert, A. Crespi, D. Ryczko, and J.-M. Cabelguen, "From swimming to walking with a salamander robot driven by a spinal cord model," Science, vol. 315, no. 5817, pp. 1416-1420, 2007.

[10] K. Seo and J.-J. E. Slotine, "Models for global synchronization in CPG-based locomotion," in Proceedings of 2007 IEEE International Conference on Robotics and Automation, Rome, Italy, April 2007, pp. 281-286.

[11] M. A. Lewis, F. Tenore, and R. Etienne-Cummings, "CPG design using inhibitory networks," in Proceedings of the 2005 IEEE International Conference on Robotics and Automation, Barcelona, Spain, April 2005, pp. $3682-3687$.

[12] S. Licht, V. Polidoro, M. Flores, F. Hover, and M. Triantafyllou, "Design and projected performance of a flapping foil AUV," IEEE Journal of Oceanic Engineering, vol. 29, no. 3, July 2004.

[13] M. Sekerli and R. J. Butera, "Oscillations in a simple neuromechanical system: Underlying mechanisms," Journal of Computational Neuroscience, vol. 19, no. 2, pp. 181-197, October 2005.

[14] Q.-C. Pham and J.-J. E. Slotine, "Stable concurrent synchronization in dynamic system networks," Neural Networks, vol. 20, no. 1, pp. 62-77, 2007.

[15] W. Wang and J.-J. E. Slotine, "On partial contraction analysis for coupled nonlinear oscillators," Biological Cybernetics, vol. 92, no. 1, pp. 38-53, 2005.

[16] D. N. Beal and P. R. Bandyopadhyay, "A harmonic model of hydrodynamic forces produced by a flapping fin," Experiments in Fluids, vol. 43, no. 5, pp. 675-682, Nonvember 2007.

[17] S. Strogatz, Nonlinear Dynamics and Chaos: With Applications to Physics, Biology, Chemistry, and Engineering (Studies in Nonlinearity). Perseus Books Group, 2001.

[18] H. K. Khalil, Nonlinear Systems, 3rd ed. Prentice Hall, 2002.

[19] S.-J. Chung and J.-J. E. Slotine, "Cooperative robot control and concurrent synchronization of lagrangian systems," IEEE Transactions on Robotics, vol. in review, 2008.

[20] M. Golubitsky, I. Stewart, P. Buono, and J. Collins, "Symmetry in locomotor central pattern generators and animal gaits," Nature, vol. 401, no. 19, pp. 693-695, October 1999.

[21] W. Lohmiller and J.-J. E. Slotine, "Contraction analysis for nonlinear systems," Automatica, vol. 34, no. 6, pp. 683-696, June 1998.

[22] A. Pikovsky, M. Rosenblum, and J. Kurths, Synchronization: A Universal Concept in Nonlinear Sciences. Cambridge University Press, 2001. 